\title{
53 MHZ FEEDFORWARD BEAM LOADING COMPENSATION IN THE FERMILAB MAIN INJECTOR *
}

\author{
J. Dey\#, I. Kourbanis, J. Reid, J. Steimel, FNAL, Batavia, IL 60510, USA
}

\section{Abstract}

$53 \mathrm{MHz}$ feedforward beam loading compensation is crucial to all operations of the Main Injector. Recently a system using a fundamental frequency down converter mixer, a digital bucket delay module and a fundamental frequency up converter mixer were used to produce a oneturn-delay feedforward signal. This signal was then combined with the low level RF signal to the cavities to cancel the transient beam induced voltage. During operation we have shown consistently over $20 \mathrm{~dB}$ reduction in side-band voltage around the fundamental frequency during Proton coalescing and over $14 \mathrm{~dB}$ in multi-batch antiproton coalescing.

\section{HARDWARE}

The purpose of the hardware (Fig. 1) is to take the Main Injector resistive wall current monitor and delay it by one turn and combine it with the $53 \mathrm{MHz}$ low level RF (VCO) fan-out sent to the cavities. This signal is down converted and up converted (In-Phase) because the Digital Bucket Delay A/D clock is operated at the VCO frequency. After the down convert, the fundamental frequency (DC component) is removed with a capacitor leaving the 90 $\mathrm{kHz}$ spaced transient mode lines to be digitized and delayed. The Digital Bucket Delay consists of an A/D converter, a FIFO, and a D/A converter that all operate off of the VCO frequency. The A/D and D/A are both 14-bit and operate between \pm 1 volt. With the FIFO, the signal is delayed by an integer numbers of rf cycles. The Main Injector harmonic number is $\mathbf{5 8 8}$ and taking into account cable delays and component positions we use the FIFO to delay the signal 531 buckets. Once delayed, the signal is cleaned up with a $30 \mathrm{MHz}$ low pass filter and up converted with the VCO. In order to run this system from 52.8 $\mathrm{MHz}$ to $53.1 \mathrm{MHz}$ up the ramp, $6 \mathrm{~ns}$ of phase shift is used of the 180 degree $53 \mathrm{MHz}$ phase shifter. A mixer was used to electronically attenuate the signal for different gains and number of bunches. A Mini-Circuits ZFSC-241124 way- $0^{\circ}$ splitter was used to fan-out the signal to each of the 18 Main Injector stations. This signal was then combined with the low level rf just after the limiter [1] in the solid state metering chassis using a MiniCircuits ZFSC-2-1 2 way combiner. In doing fan-out and fan-back for Feedforward BLC, one must take into account the time of flight for the particles to pass each station. The fan-back also uses the Mini-Circuits ZFSC-24-11 to vectorialy sum up all of the Main Injector Cavity gap monitors. Since the Pbars spin in the opposite direction, Station 18 now becomes the first cavity, a whole separate time of flight fan-out and fan-back system was created for Pbar Feedforward BLC.

\section{OPERATION}

Feedforward (FF) Beam Loading Compensation (BLC) was first done during Proton Coalescing at $150 \mathrm{GeV}$. Later, it was also implemented during Pbar Coalescing at $150 \mathrm{GeV}$. Presently we are in the process of commissioning Feedforward BLC to work up the Ramp from $8 \mathrm{GeV}$ to $150 \mathrm{GeV}$.

\section{Proton Coalescing}

Figure 2 shows a Mountain Range of typical seven bunch Proton Coalescing using both feedforward and feedback [1] BLC. The HP 89441A Vector Signal Analyzer (VSA) plot (Fig. 3) at $150 \mathrm{GeV}$ shows a reduction in the transients by $26 \mathrm{~dB}$. The VSA plot was made from the fan-back of all eighteen stations. In adding feedforward BLC, Proton Coalescing efficiency improved by $5 \%$ and day-to-day reliability has been greatly enhanced.

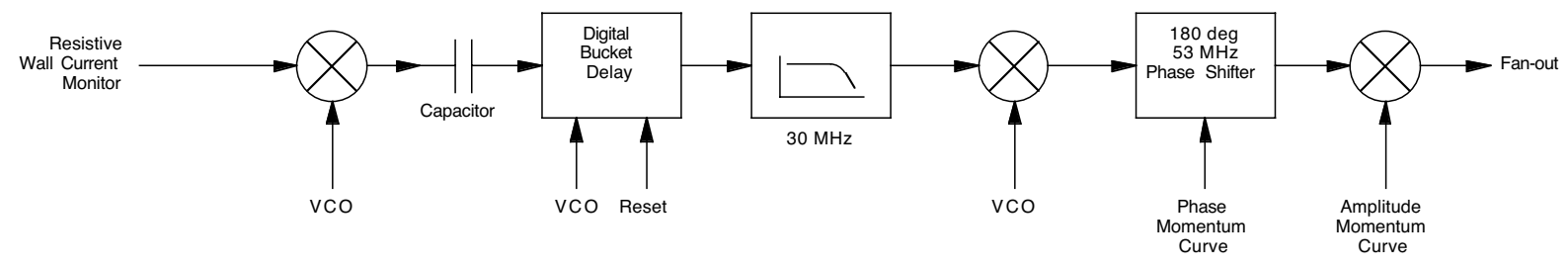

Figure 1: Block Diagram of Feedforward Beam Loading Compensation 

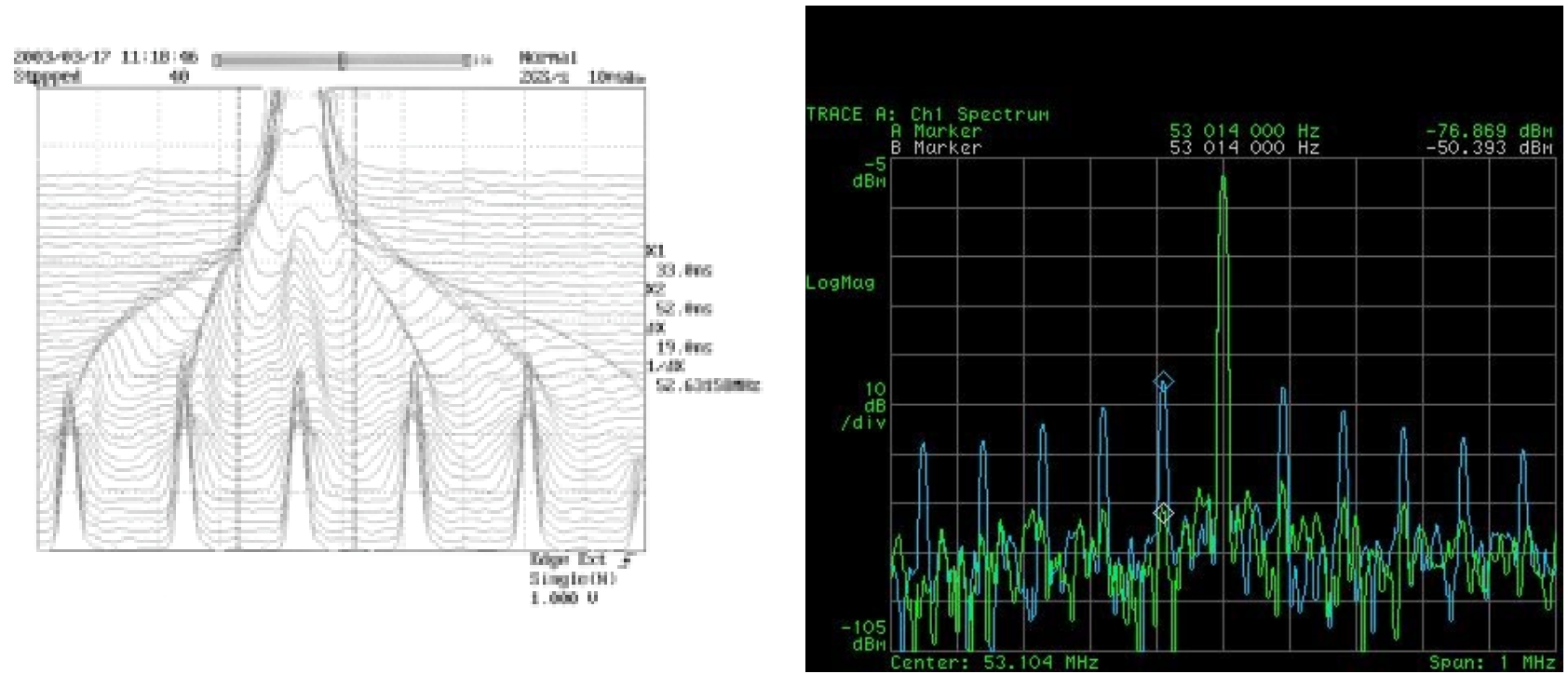

Figure 2: Proton Coalescing of 283E9 Protons

\section{Pbar Coalescing}

Pbar Coalescing consists of multi-bunch coalescing. Each of the four bunches only consists of 30E9 Pbars so intensity is not the issue here but alignment during recapture of all four bunches is. Figure 4 shows a reduction in the transient modes of $15 \mathrm{~dB}$. One can clearly see in Figure 5 the multi-bunch alignment problem that occurs with feedback BLC only being applied. In Figure 6 , the coalesced beam was recaptured properly and no beam appears outside the bucket on the right side when both Feedback and Feedforward BLC are applied. Overall Pbar Coalescing efficiency rose from $75 \%$ to $87 \%$ because of this improvement.

\section{Feedforward BLC up the Ramp}

Early promising results are shown for FF BLC up the Ramp. A Merrimac PMP-3R-53B 180 degree $53 \mathrm{MHz}$ phase shifter is used to track the $6 \mathrm{~ns}$ time of flight difference from $8 \mathrm{GeV}$ to $150 \mathrm{GeV}$. The difference of only $6 \mathrm{~ns}$ is attributed to the 531 buckets of delay that is removed by the Digital Bucket Delay being clocked off the VCO. A mixer was used after the phase shifter to

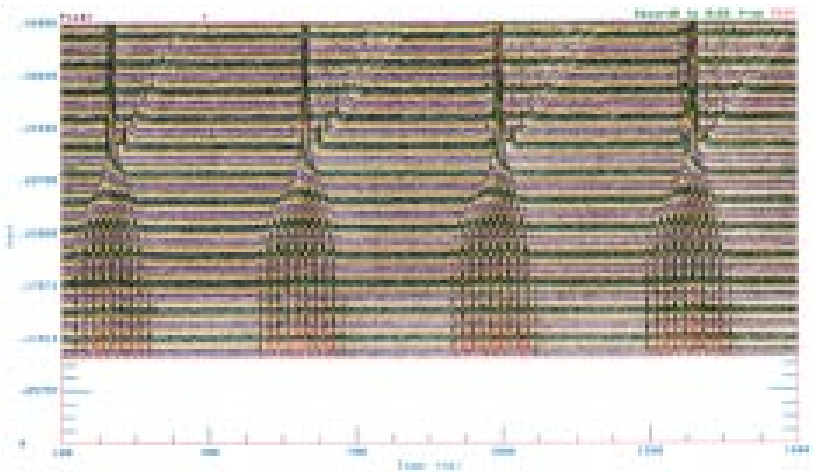

Figure 5: Without FF BLC on Pbar Coalescing

Figure 3: Main Injector Cavity Gap Response with (green) and without (blue) Feedforward BLC during Proton Coalescing

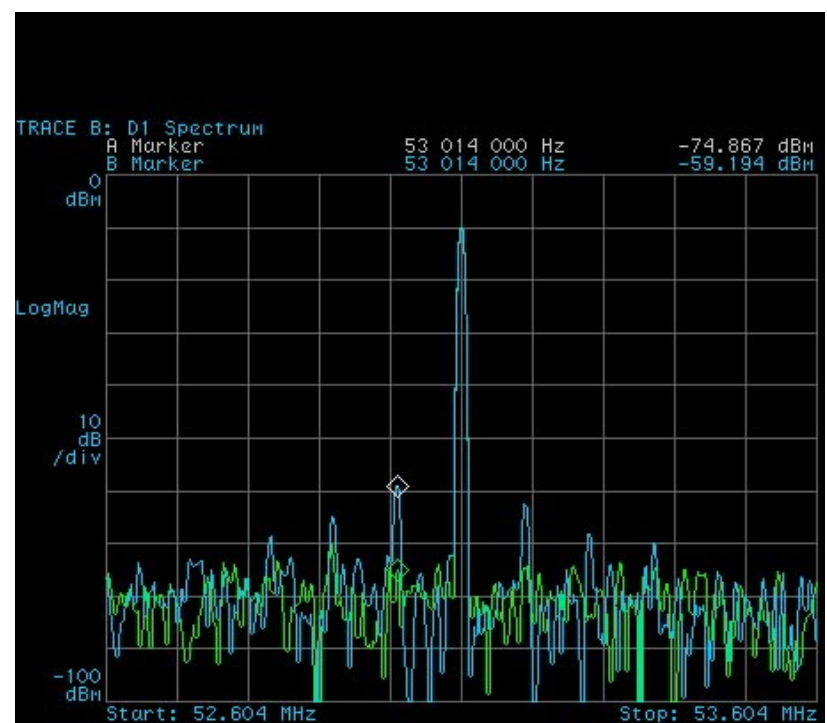

Figure 4: Main Injector Cavity Gap Response with (green) and without (blue) Feedforward BLC during Pbar Coalescing

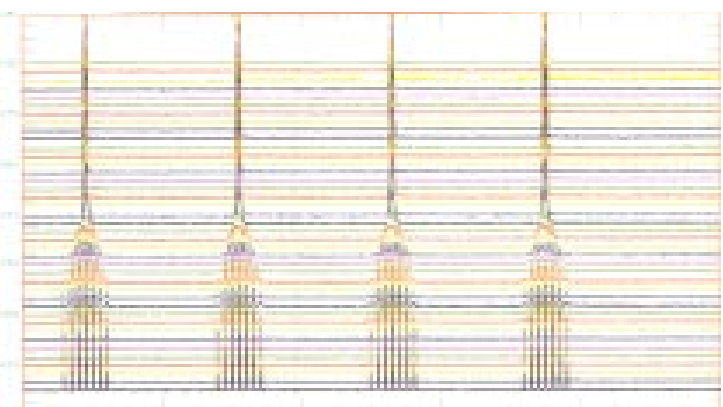

Figure 6: With FF BLC on Pbar Coalescing 


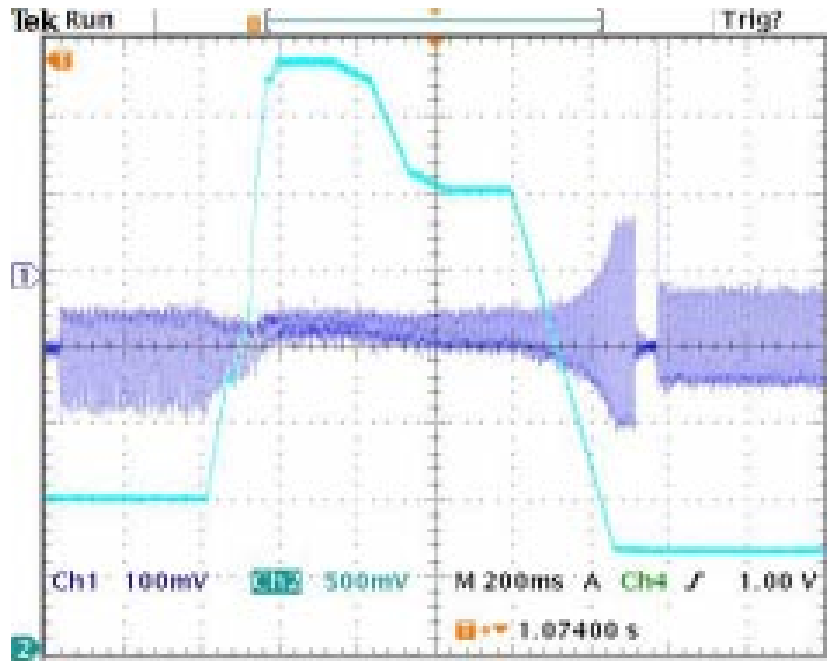

Figur\%7: Without F\%alforward BLC up th\%Ramp

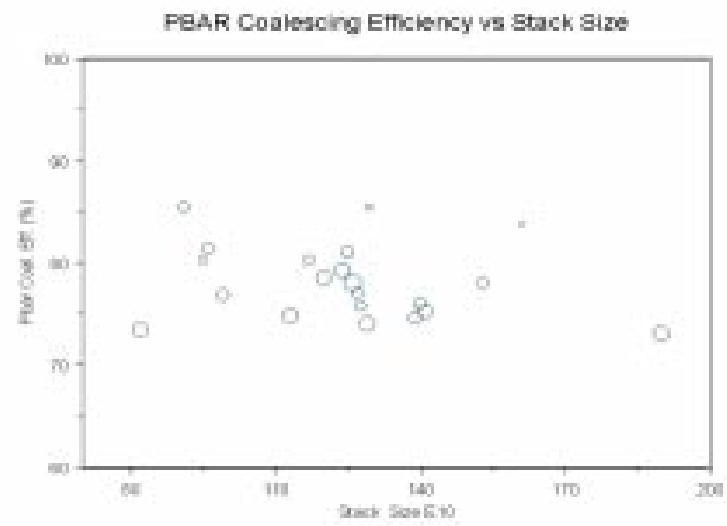

Figure 9: Coalescing without Feedforward BLC

electronically attenuate the FF BLC for different gains up the ramp. Figure 7 and 8 show the Local Station Phase Detector $\left(0.9^{\circ} / \mathrm{div}\right)$ response (blue trace) and the Detected RF Gap Envelope (aqua trace) for Main Injector Station \#1 during Proton Coalescing. We also plan to use FF BLC up the Ramp during a Main Injector Pbar Stacking Cycle were it is expected to help us reduce the longitudinal emittance blow-up (especially through transition.)

\section{RESULTS}

Figure 9 shows the average Pbar Coalescing Efficiency as a function of Stack Size without FF BLC. The size of

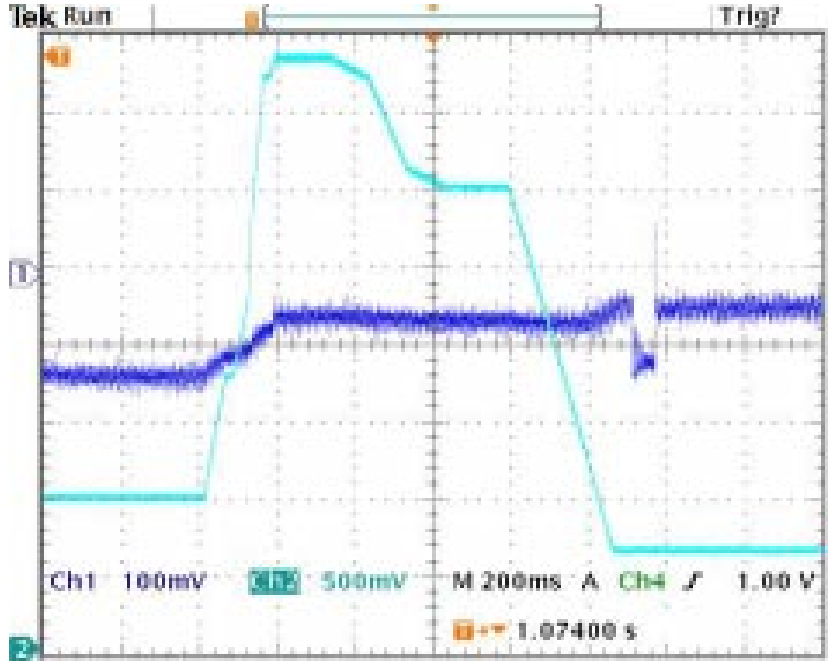

Figure 8: With Feedforward BLC up the Ramp

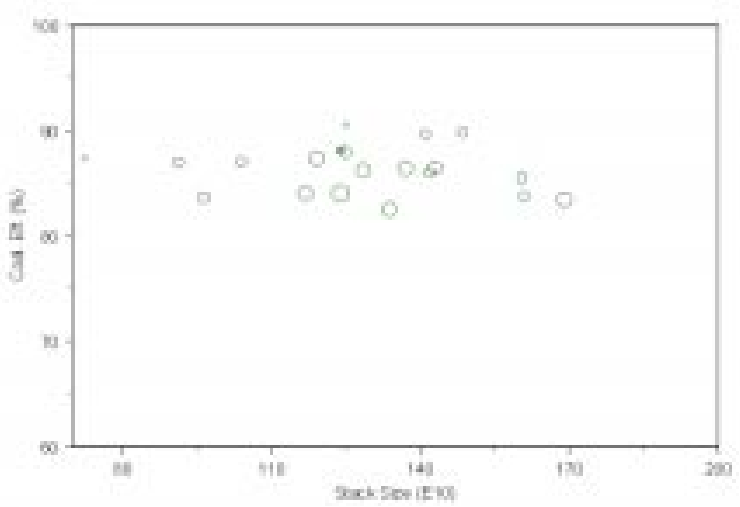

Figure 10: Coalescing with Feedforward BLC

the circles is proportional to the longitudinal emittance of the Pbar bunches before coalescing. Average Coalescing efficiency was about $75 \%$ and was getting lower with the larger stack size. Figure 10 is with Feedforward BLC. The average Coalescing efficiency was about $87 \%$ and no dependence with stack size is observed.

\section{REFERENCES}

[1] J. D\%, J. St\%m\%, J. R\%d, "Narrowband B\%m Loading Comp\%sation in th\% $\%$ \%milab Main Inj\%tor Acc\%\%ating Caviti\%," 2001 PAC, p. 876, Chicago, Jun\%2001. 